\title{
Stage IVB Appendix Carcinoma AJCC v7
}

National Cancer Institute

\section{Source}

National Cancer Institute. Stage IVB Appendix Carcinoma A/CC v7. NCI Thesaurus. Code C87807.

Stage IVB includes: (Any T, N0, M1a, G2, G3); (Any T, N1, M1a, Any G); (Any T, N2, M1a, Any G). N0: No regional lymph node metastasis. N1: Metastasis in 1-3 regional lymph nodes. N2: Metastasis in four or more regional lymph nodes. M1a: Intraperitoneal metastasis beyond the right lower quadrant, including pseudomyxoma peritonei. G2: Moderately differentiated tumor. G3: Poorly differentiated tumor. (AJCC 7th ed.) 